\title{
Structure elucidation by LC-MS. Foreword
}

\author{
W.M.A. Niessen \\ hyphen MassSpec Consultancy, De Wetstraat 8, 2332 XT Leiden, The Netherlands
}

\section{Introduction}

The topic of this Analusis Dossier is structure elucidation by liquid chromatography - mass spectrometry (LC-MS). When LC-MS first came along in the mid 1970's [1], one of the incentives and objectives was to develop a technique similar to the very successful gas chromatography - mass spectrometry (GC-MS), but capable of identifying especially those components which were not amenable to GC-MS. To that end, interfaces were developed, aiming at the application of electron ionisation (EI), like in GC-MS. Although these interfaces like the moving-belt and the particle-beam interface actually demonstrated that the applicability range of EI could be significantly extended, ironically enough these two interfaces did not achieved the major breakthroughs in the development of LC-MS.

Major steps in the history of LC-MS were made using interfaces that only allow soft ionisation techniques, resulting in (de)protonated molecules with little or no fragmentation. Thermospray interfacing from the mid 1980 onwards for the first time gave a glimpse on what LC-MS could really do. With the broad implementation of the atmospheric-pressure ionization and interfacing, viz. (pneumatically-assisted) electrospray ionisation and interfacing (ESI) and the heated nebuliser in combination with atmosphericpressure chemical ionisation (APCI), LC-MS became a powerful technique which could even be used by less experienced people. However, in addition, the most frequent use of LC-MS today is quite different from the initial objective indicated above. LC-MS has significantly changed the impact of MS in a laboratory, because with LC-MS the mass spectrometer has entered both laboratories and application areas in a way which was certainly not foreseen at the beginning of its history in the mid 1970's [2]. Especially the large interest in routine quantitative analysis and peptide and protein analysis was not anticipated.

With LC-MS being based on soft ionisation techniques, the development of LC-MS to some extent stimulated the developments in tandem mass spectrometry, especially in triple-quadrupole and ion-trap instruments. With the ability to fragment the even-electron protonated or deprotonated ions, generated in ESI and APCI, by means of collisioninduced dissociation (CID), the first steps in the direction to structure elucidation could be made. However, soon it was realised that in fact there is far less knowledge on the fragmentation of these even-electron ions compared to that of odd-electron ions [3], as generated in EI. Despite the fact that many authors tried and still try to cover this by incorrectly calling the (de)protonated molecule a "(de)protonated molecular ion' [4], this difference is obvious and important when one starts to interpret the MS-MS product-ion mass spectra. In addition, the interpretation of the product-ion mass spectra must actually be performed, because no spectral libraries were available which could be used to assist in the structure elucidation.

\section{Fragmentation of even-electron ions}

In the past 14 years, ever since my practical introduction to MS-MS in 1986, the editor of this Dossier has developed a great interest in the interpretation of MS-MS product-ion mass spectra generated from the dissociation of even-electron ions. Like many colleagues in the field, I was often puzzled by the fragmentation observed. Sometimes, a useful strategy appeared to be: just cut the molecule at the bonds which are likely to be cleaved, play a little with proton shifts in order to get the correct $\mathrm{m} / \mathrm{z}$ for the fragment, but do not pay much attention to the actual fragmentation mechanism. From a pragmatic point of view, this can be quite a successful approach, but certainly not always, and also often both frustrating and unsatisfactory.

By studying many MS-MS product-ion mass spectra of a wide variety of molecules, one starts to get a better insight.

$$
\begin{aligned}
& \text { Ether } \underset{\mathrm{H}^{+}}{\stackrel{\mathrm{R}-\mathrm{C}_{\mathrm{O}} \mathrm{C}}{\mathrm{H}_{\mathrm{O}}}} \longrightarrow \underset{\mathrm{R}}{\mathrm{H}-\mathrm{O}-\mathrm{R}} \mathrm{H}^{+} \text {or } \mathrm{R}-\mathrm{C}=\mathrm{C}^{+} \\
& \text {Amine } \underset{\mathrm{H}^{+}}{\stackrel{\mathrm{R}}{\mathrm{H}}-\mathrm{C}-\mathrm{R}} \longrightarrow \underset{\mathrm{H}-\mathrm{N}-\mathrm{R}}{\mathrm{H}-\mathrm{H}} \text { or } \mathrm{R}-\mathrm{C}=\mathrm{C}^{+}
\end{aligned}
$$

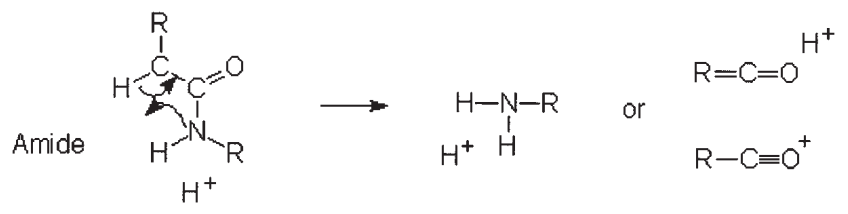

Figure 1. Four-center fragmentation mechanism, illustrated for ethers, amines and amides. 
Some major fragmentation reactions of a variety of compound classes can be rationalised by means of a four-center mechanism, as demonstrated in figure 1. However, especially in the last few years, several detailed studies were published on the actual fragmentation mechanisms of some protonated molecules, using derivatisation, isotope labelling, precursorion experiments, accurate mass determination, and other advanced MS methods. Some examples are related to the fragmentation of testosterone [5], propanolol [6], and polyamine spider toxins [7]. In fact, one of these studies [7] indicated that the four-center mechanism, though useful in the rationalisation of some fragmentation reactions, is actually not the correct mechanism for $N$-(4aminobutyl)-3-(4hydroxyphenyl)prop-2-enamide. Deuterium labelling experiments showed that the fragmentation in this case can better be explained from neighbouring-group participation (see Fig. 2).

Fragmentation of even-electron negative-ions is generally considered to be even more complex than that of positive ions. However, the main fragmentation routes in the fragmentation of negative-ions are well classified by Bowie [8]. These fragmentation routes, some of which are indeed

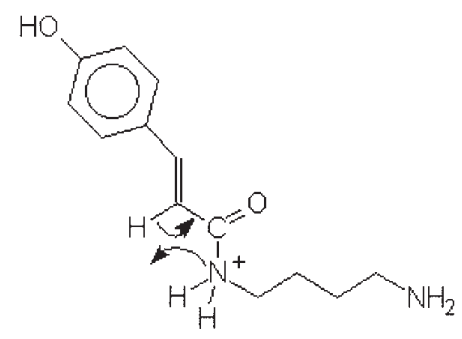

(a)

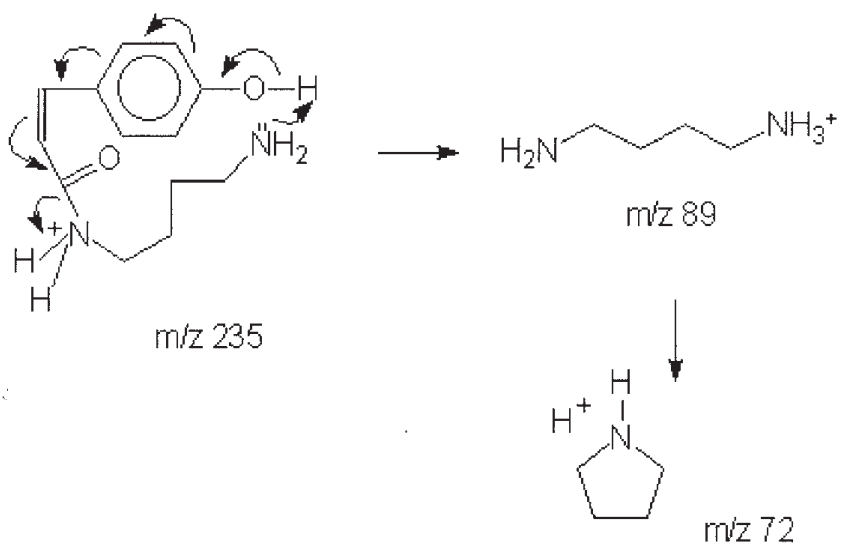

(b)

Figure 2. Fragmentation of the polyamine spider toxin $\mathrm{N}$ (4aminobutyl)-3-(4-hydroxyphenyl)prop-2-enamide via (a) fourcenter fragmentation and (b) neighbouring-group participation mechanisms (Based on ref. [7]). complex, can actually be applied to explain the most important dissociations in negative-ion MS-MS, as is for instance demonstrated in studies related to the fragmentation of ethylene glycol diacetates [9] and poly-hydroxy acids [10].

\section{Progress in mass spectrometry instrumentation}

Developments in instrumentation also facilitate more advanced studies in fragmentation and mechanisms. An example is the current availability of routinely applicable micro- and nano-LC instrumentation, which more readily enables the use of deuterated solvents, for instance, to perform $\mathrm{H} / \mathrm{D}$ exchange studies, e.g., for discrimination between $\mathrm{N}$-oxides and hydroxylated compounds [11].

A powerful tool in elucidating fragmentation mechanisms is the ion trap, which allows step-wise and controlled fragmentation in multiple-stage MS-MS. A nice and recent example of its potential is demonstrated in the elucidation of the fragmentation pattern of dextromethorphan and some of its metabolites [12].

The ability of a quadrupole-time-of-flight hybrid instrument (Q-TOF) to provide accurate mass determination of the product ions in MS-MS is yet another powerful tool in structure elucidation, not so much in elucidation of the mechanism, but in understanding the fragments that are actually generated. An elegant example of the application of the Q-TOF is described by Hopfgartner et al. [13] for bosentan and its metabolites.

Some examples of the combined use of ion-trap and/or Q-TOF instruments in structure elucidation are given in this Dossier.

\section{Towards routine structure elucidation}

This Analusis Dossier contains a collection of papers demonstrating the significant progress that is made in structure elucidation by LC-MS and typical strategies applied in this area.

Any structure elucidation by MS starts with a proper look at the mass spectrum of the compound of interest prior to its fragmentation. Attention should be paid to adduct ions and isotope peaks. For some compounds a combination of positive-ion and the negative-ion mode can be used to obtain a better confidence concerning the molecular mass of the unknown. Such data provide useful information on the compound of interest.

Fragmentation of the (de)protonated molecule can be achieved in a variety of ways: (1) by in-source CID via an increase of the potential difference between the ion-sampling aperture and the skimmer (in most instruments), (2) by lowenergy collisions in a collision cell of a triple-quadrupole or 
a Q-TOF instrument, (3) by low-energy collisions in an ion trap, and (4) by high- or low-energy collisions in the fieldfree regions of a double-focussing sector instrument. Examples of all these approaches are available in this Dossier.

Rationalisation of fragmentation reactions, sometimes even without a thorough study of the underlying mechanisms, can be useful to get better insight in the type of fragmentations that are involved. Subsequently, detailed studies using labelled components and advanced strategies must be performed to gradually elucidate the actual mechanisms. One should not forget that the extensive studies of fragmentation mechanisms with relatively simple components, as performed since many years by means of EI [3], are actually the basis for our current understanding of the fragmentation in EI and for our ability to, at least partly, identify real unknowns by EI-MS. Therefore, the study into the fragmentation mechanism and structure-fragmentation relation will in the end be extremely important.

However, we cannot wait until much more progress has been made in such studies. We have to elucidation (partially) unknown structures already today. We can take advantages of powerful tools, such as ion-trap and Q-TOF instruments. But also, we see a great interest in building CID spectral libraries and explorations of its potential use. Examples of such an approach to structure elucidation are included in this dossier as well.

Structure elucidation is important in almost all fields were MS is applied. This is demonstrated by the various application areas covered in this Dossier: drug metabolites, natural products, pesticides, steroids, compounds of toxicological interest. The papers in this Dossier were deliberately focussed on the structure elucidation of relatively small molecules (typically below $700 \mathrm{Da}$ ). No attention is paid to identification and structure elucidation of biomacromolecules. We hope that this Dossier is useful for researchers involved in structure elucidation by means of LC-MS technologies, and that it will further stimulate detailed and fundamental research efforts in the area.

\section{References}

1. Niessen, W.M.A., Liquid Chromatography - Mass Spectrometry, 2nd Ed., 1999, Marcel Dekker Inc., New York, NY.

2. Niessen, W.M.A., J. Chromatogr. A 1999, 856, 179.

3. McLafferty, F.W.; Turček, F. Interpretation of Mass Spectra; Mill Valley, CA.: University Science Books, 4th Ed., 1993.

4. Bursey, M.M. Mass Spectrom. Rev. 1991, 10, 1.

5. Williams, T.M.; Kind, A.J.; Houghton E.; Hill, D.W. J. Mass Spectrom. 1999, 34, 206.

6. Upthagrove, A.T.; Hackett M.; Nelson, W.L. Rapid Commun. Mass Spectrom., 1999, 13, 534.

7. Bigler L.; Hesse, M. J. Am. Soc. Mass Spectrom. 1995, 6, 634.

8. Bowie, J.H. Mass Spectrom. Rev. 1990, 9, 349.

9. Stroobant, V.; Rozenberg, R.; Bouabsa, E.M.; Deffense E.; de Hoffmann, E. J. Am. Soc. Mass Spectrom. 1995, 6, 498.

10. Wheelan, P.; Zirrolli J.A.; Murphy, R.C. J. Am. Soc. Mass Spectrom. 1996, 7, 140.

11. Ohashi, N.; Furuuchi S.; Yoshikawa, M. J. Pharm. Biomed. Anal. 1998, 18, 325.

12. Strife, R.J.; Robosky, L.C.; Garrett, G.; Ketcha, M.M.; Shaffer J.D.; Zhang, N. Rapid Commun. Mass Spectrom. 2000, 14, 250.

13. Hopfgartner, G.; Chernushevich, I.V.; Covey, T.; Plomley J.B.; Bonner, R.; J. Mass Spectrom. 1999, 10, 1305. 